\title{
Novel implementation of a Kalman filter for speckle nulling with a fiber injection unit
}

Manxuan Zhang, Jorge Llop Sayson, Nemanja Jovanovic, Dimitri Mawet, Yeyuan Xin

Manxuan Zhang, Jorge Llop Sayson, Nemanja Jovanovic, Dimitri Mawet, Yeyuan Xin, "Novel implementation of a Kalman filter for speckle nulling with a fiber injection unit," Proc. SPIE 11117, Techniques and Instrumentation for Detection of Exoplanets IX, 111171X (10 September 2019); doi:

$10.1117 / 12.2530756$

Event: SPIE Optical Engineering + Applications, 2019, San Diego, California, United States 


\title{
Novel implementation of a Kalman filter for speckle nulling with a fiber injection unit
}

\author{
Manxuan Zhang ${ }^{\mathrm{a}}$, Jorge Llop Sayson ${ }^{\mathrm{a}}$, Nemanja Jovanovic ${ }^{\mathrm{a}}$, Dimitri Mawet ${ }^{\mathrm{a}, \mathrm{b}}$, and Yeyuan \\ $\mathrm{Xin}^{\mathrm{c}}$ \\ ${ }^{a}$ Department of Astronomy, California Institute of Technology, 1200 E. California Blvd., \\ Pasadena, USA, 91125 \\ bJet Propulsion Laboratory, California Institute of Technology, 4800 Oak Grove Dr., Pasadena, \\ CA 91109, USA \\ ${ }^{\mathrm{c}}$ Department of Aeronautics and Astronautics, Massachusetts Institute of Technology, 125 \\ Massachusetts Ave., Cambridge, MA 02139
}

\begin{abstract}
High dispersion coronagraphy (HDC) is a technique that combines high contrast imaging techniques with high spectral resolution spectroscopy to directly characterize exoplanets and provide key information such as chemical composition, temperature, and rotational velocity. A consequence of adaptive optics systems used in direct imaging is the formation of residual bright spots of star lights, called speckles, in the final image. Due to the large difference in brightness between host stars and their planets, these speckles can easily obscure potential exoplanets. In a previous demonstration, it was shown that using monochromatic light and a fiber injection unit (FIU), simulated exoplanet light can be directed to a high-resolution spectrograph. The method had speckle suppression that exceeding conventional image-based speckle nulling. With a previous Kalman filter estimator implementation, we found that with the implementation of the algorithm, speckle suppression was even more stable and outperformed traditional speckle nulling. In this update to the estimator, progress has been made in terms of a new filter design, and better estimates of the physical parameters in the laboratory, resulting in a higher speckle nulling performance.
\end{abstract}

Keywords: Vortex Coronagraph, Fiber Injection Unit, Speckle Nulling, Kalman Filter, Spectrograph, Exoplanet Characterization, Starlight-Suppression, High-Contrast Imaging

\section{INTRODUCTION}

Direct imaging is a technique in exoplanet detection that can be used to gather more identifying characteristics of planetary companions. These characteristics such as temperature, chemical composition, and spin information, can all be particularly useful in determining habitability. This technique does come with a set of challenges, of which include the limitations of adaptive optics (AO) systems. Though AO systems use a variety of different methods to sense and correct wavefronts, leftover effects caused by non-common path aberration (NCPA) - which can come from slow acting changes to the optics system and also disturbances from turbulence and vibration - cause imperfect coronagraphic starlight suppression. As a result of this imperfect suppression, bright spots of starlight manage to leak through to the final image. This is problematic as these speckles display very similar characteristics to planets, such as their apparent size and brightness. A large field of speckles can easily suppress the signal from actual planetary companions. Even without the problem of residual speckles from the coronagraph, the large contrast in the brightness of the star and any of its companions would inevitably cause residual starlight to completely obscure any planet light.

With coronagraphs, much better contrast can be achieved. However, coronagraphs are very sensitive to uncorrected residual aberrations, and thus are often not working at maximum efficiency. One attempt to supplement the starlight suppression capabilities of the coronagraph is to employ a fiber injection unit (FIU). ${ }^{1}$ In high

Further author information: (Send correspondence to Manxuan Zhang)

Manxuan Zhang: E-mail: mzhang3@caltech.edu

Techniques and Instrumentation for Detection of Exoplanets IX, edited by Stuart B. Shaklan, Proc. of SPIE Vol. 11117, 111171X · @ 2019 SPIE · CCC code: 0277-786X/19/\$21 - doi: 10.1117/12.2530756 
dispersion coronagraphy, (as illustrated in figure 1) a high contrast instrument (including an AO or wavefront control system and a coronagraph) would be linked via a FIU to a high-resolution spectrograph. Raw data (including signal from the planet, residual starlight, and noise sources such as background noise, readout noise, and photon shot noise) would be cross-correlated with a theoretical template to create a cross-correlation function $(\mathrm{CCF}) .{ }^{1}$ The profile of the CCF would allow for improved dynamic range, and also allow for detection of molecular characterization, spin measurements, and Doppler imaging. ${ }^{1}$ High-spectral-resolution observations would also render spatial speckle noise irrelevant. ${ }^{1}$ This is because the spectral signature of the stellar speckle is very smooth, and does not vary rapidly with wavelength. ${ }^{1}$ As a result, it becomes part of the continuum at very high spectral resolutions, and speckle noise would be drastically reduced. ${ }^{1}$ The FIU also provides multiple degrees of freedom that help with nulling residual starlight that couples into the fiber, with the injection efficiency of the FIU being given by:

$$
\eta=\frac{\left|\iint H E_{11}(x, y) A^{*}(x, y) d x d y\right|^{2}}{\iint\left|H E_{11}(x, y)\right|^{2} d x d y \iint|A(x, y)|^{2} d x d y}
$$

As asymmetric fields would not couple into the fiber, this allows for even greater contrast gains in starlight suppression. ${ }^{1}$

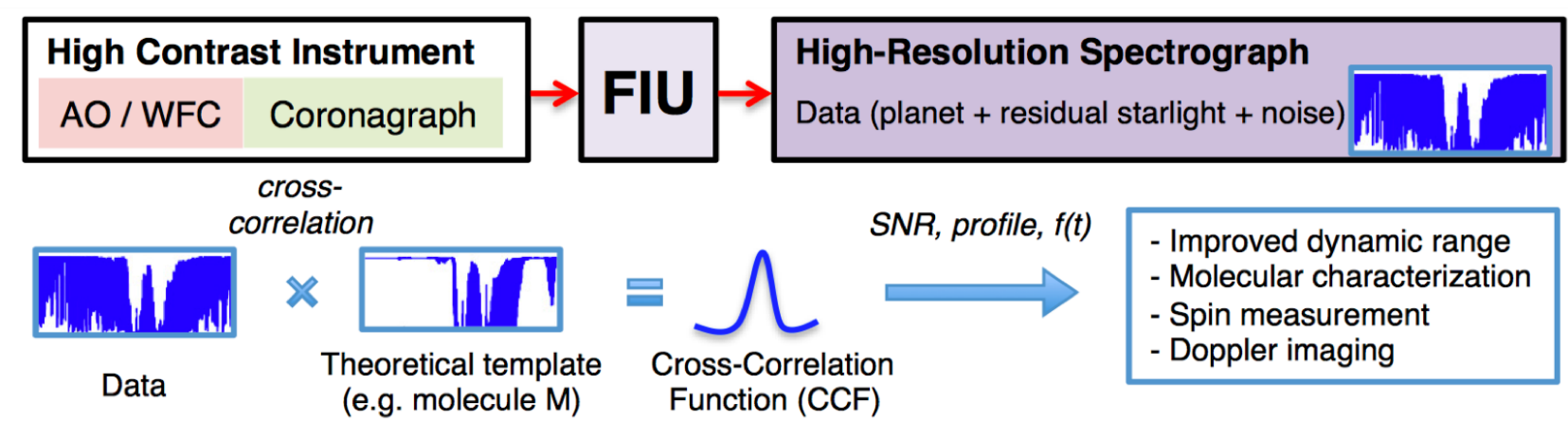

Figure 1. Diagram illustrating the concept for high dispersion coronagraphy (HDC). HDC combines high contrast imaging techniques using coronagraphy with high spectral resolution spectroscopy, and its successful implementation is a critical step towards understanding exoplanet atmospheres. ${ }^{1}$

\section{LABORATORY SETUP}

The High Contrast Spectroscopy Testbed (HCST) at the Caltech Exoplanet Technology Lab is a facility with the goal of testing high contrast imaging and spectroscopy technologies for future applications. ${ }^{2}$ Figure 2 shows the set-up of the transmissive bench of HCST, which consists of a telescope simulator, an AO system, a coronagraph, and an FIU. The star and an off-axis companion are simulated by two different sources, a $635 \mathrm{~nm}$ laser diode and a narrow band filtered (at $650 \mathrm{~nm}$ ) supercontinuum white light source respectively. The telescope simulator images these two sources. The AO system consists of a 144-actuator MEMS DM, a beam splitter to divert some light for wavefront sensing, and a Shack-Hartmann wavefront sensor.

The AO system is followed by a relay of a focusing lens, a charge 4 vortex focal-plane mask (50\% off-axis transmission at $1.7 \lambda / D)$, collimating lenses, and a Lyot stop. The focal-plane mask is phase-based and rejects light using a phase singularity at the center of the device that diffracts light outwards. This diffracted light is blocked off by a Lyot stop.

Downstream of the Lyot stop is the FIU, which is shown in figure 3. It consists of a three-axis tip-tilt mirror controlled by piezoactuators, allowing the beam to be steered into the single mode fiber. It also contains a dichroic that allows some light to be split off and sent to a tracking camera. The single-mode fiber can be used to back-propagate the light for calibration purposes. This particular FIU had been demonstrated to be able to successfully couple monochromatic light from the simulated exoplanet source and direct it towards a 
high-resolution spectrograph. For the demonstration purposes of this experiment, a powermeter was used in place of where a spectrograph would be for science observations.

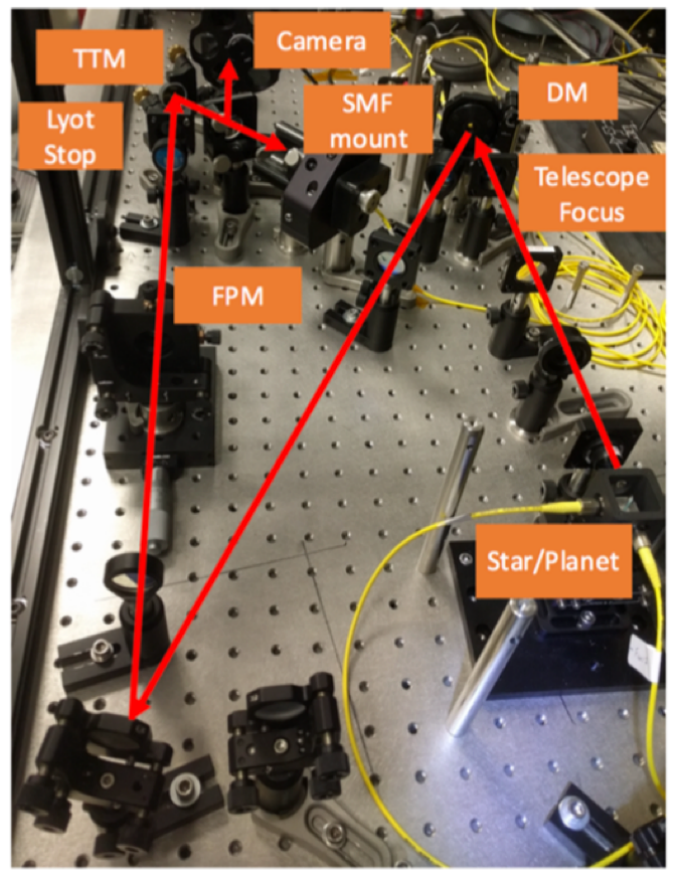

Figure 2. The layout of the transmissive bench of HCST. Many preliminary demonstrations in Cal- Fig tech's Exoplanet Technology lab are conducted tory demonstration to direct monochromatic light into a powermeon this testbed.

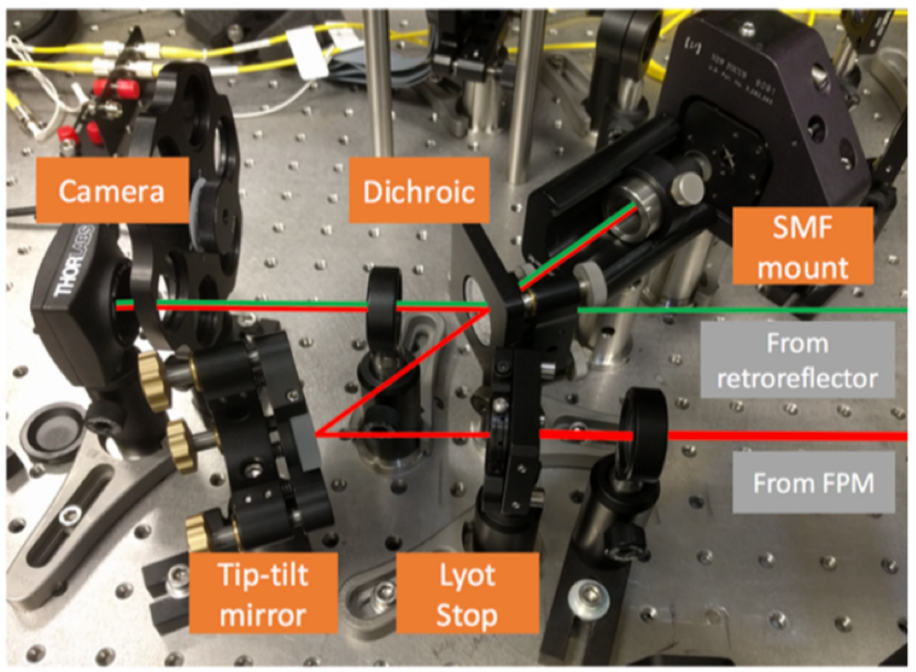

ter.

\section{SPECKLE NULLING}

\subsection{Variable Definition}

Speckle nulling attempts to remove bright speckles in the final image by creating an anti-speckle and using destructive interference. In this particular case, a single sinusoid would be applied to the DM, and the parameters of the sinusoid (amplitude, phase, period etc.) would be tweaked in order to find the anti-speckle that produces the best null. In each iteration, we apply a cosine pattern to the DM with the amplitude:

$$
h=\frac{h_{0}}{4}[\cos (2 \pi \vec{\xi} \cdot \vec{r}+\alpha)]
$$

Here, $h_{0} / 2$ is the maximum possible height of a single actuator $\left(h_{0} / 4\right.$ being half of the maximum height), $\vec{\xi}$ is the spatial frequency vector which indicates the location of the speckle in the image plane, $\vec{\xi}$ indicates the position of the speckle in the pupil plane, and $\alpha$ indicates a constant phase offset. Thus, with these four variables, the intensity, position, and phase of the speckle can be defined.

\subsection{Calibration}

In order to determine the correspondence between the parameters of the sinusoid when applied to the DM and its effects on the speckle, a calibration sequence must be run before each speckle nulling experiment. A series of parameters (different spatial frequencies, intensities, and phase) would be applied to the DM and using the camera, the position of the speckle would be measured and $\vec{\xi}$ would be determined.

In order to determine the correspondence between the applied height of DM actuators and the effect on the speckle's intensity when coupled through the fiber, the speckle's location can be held constant with four different 
phases tested (in order to account for the effects of the phase), while the amplitude is changed and the effect of the change on throughput is measured using the powermeter.

The results of the amplitude, phase, and spatial frequency calibrations are saved in lookup tables that would be used during speckle nulling.

\subsection{Nulling}

In order to null a speckle that is on the fiber, the location would first be determined from the camera. From there, the appropriate $\vec{\xi}$ value to produce an anti-speckle at the same location would be determined from the calibration data. After that, a test or "probe" speckle is placed at the location of the fiber. Using a DM amplitude that is much less than the measured amplitude of the speckle, power readings are taken at four different phases. By taking the Fourier transform of the four different readings and using the calibration tables, the $h_{0}$ and $\alpha$ that create the anti-speckle that best nulls the speckle would be determined. ${ }^{3}$ This process is repeated iteratively: after the best anti-speckle is applied to the DM, the procedure of probing and re-applying the new best anti-speckle is repeated.

\section{KALMAN FILTER ESTIMATOR}

A Kalman filter estimator uses control history and continual measurements in order to obtain better and more stable estimates of the speckle parameters and thus better nulling with better anti-speckles. Below, we go through the architecture of the Kalman filter used in this experiment.

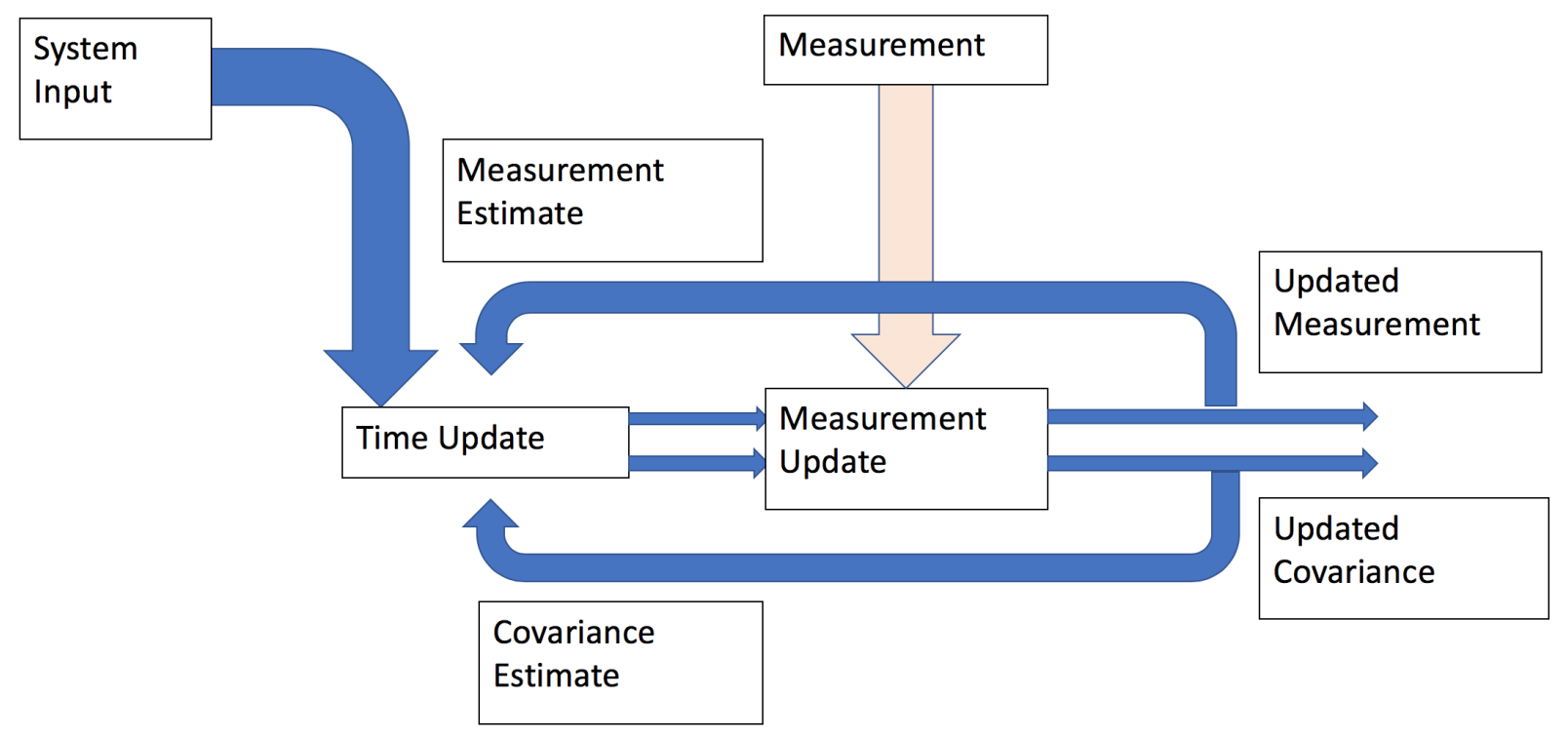

Figure 4. Diagram illustrating the general concept of a Kalman filter. Before each measurement, a best guess based on previous measurements is made. After the measurement is taken, the best guess is updated and a new covariance is calculated. The final calculated best output is given to the DM. The cycle repeats iteratively while nulling is happening.

\subsection{Filter Variables}

The following is a description and explanation of the variables shown in table 1 .

1. Propagation Matrix: Relates the control input to its actual effect on the state

2. Linear Observation Matrix: Relates the measured state to the actual state 
Table 1. Definition of all matrices in the Kalman filter architecture

\begin{tabular}{|l|l|}
\hline Definition & Function \\
\hline$\Phi=I$ & State Transition Matrix \\
\hline$\Gamma=I$ & Propagation Matrix \\
\hline$H_{k}=I$ & Linear Observation Matrix \\
\hline$z_{k}=\left(\begin{array}{c}\psi \\
A\end{array}\right)$ & Measurement \\
\hline$z_{k}=\left(\begin{array}{c}\psi \\
A\end{array}\right)$ & Estimation \\
\hline$u=\left(\begin{array}{c}\phi \\
h_{0}\end{array}\right)$ & Control Input \\
\hline$P_{0}=\left(\begin{array}{cc}\varphi_{\phi}^{2} & 0 \\
0 & \sigma_{A_{i}}^{2}\end{array}\right)$ & Initial Covariance Matrix \\
\hline$Q=\left(\begin{array}{cc}\sigma_{\phi}^{2} & 0 \\
0 & \sigma_{A_{p}}^{2}\end{array}\right)$ & Process Noise Matrix \\
\hline$R=\left(\begin{array}{cc}\sigma_{\phi_{m}}^{2} & 0 \\
0 & \sigma_{A_{m}}^{2}\end{array}\right)$ & Measurement Noise Matrix \\
\hline
\end{tabular}

3. Measurement: Measurement of the phase and amplitude of the speckle

4. Estimation: Estimation of the phase and amplitude of the speckle

5. Control Input: The actual input used to attempt to null the speckle, which can also be represented as:

$$
u_{k}=\left(\begin{array}{c}
\phi \\
h_{0}
\end{array}\right)=-\left(\begin{array}{c}
\hat{\psi} \\
\hat{A}
\end{array}\right)=-\hat{x}_{k}(+)
$$

6. Initial Covariance Matrix: Includes the uncertainties on phase and amplitude on the initial state guesses

7. Expected Process Noise: We assume that it is dominated by DM control error ${ }^{4}$

8. Expected Measurement Noise: Noise from sensors, including dark noise, read noise, and photon shot noise ${ }^{4}$

\subsection{Process}

For each iteration, this is the process used to determine the best solution for the anti-speckle:

1. A best guess at the state before measurement is made based on previous control history, using the state transition matrix and propagated control input

2. The error covariance of the best guess at the state before the measurement is determined

3. A measurement is taken

4. The best guess is updated given the measurement information

5. The error covariance of the updated best guess is determined

6. The control input based on the updated best guess is applied

7. The process is repeated

The standard Kalman filter equations corresponding to the steps described above are given in Table 2. Below, the state is given by $\hat{x}_{k}$, the covariance is given by $P_{k}$, and the gain is given by $K_{k}$, for each $k^{t h}$ iteration. ${ }^{4}$ 
Table 2. Definition of all matrices in the Kalman filter architecture

\begin{tabular}{|l|l|}
\hline Equation & Function \\
\hline$\hat{x}_{k}(-)=\Phi_{k-1} \hat{x}_{k-1}(+)+\Gamma_{k-1} u_{k-1}$ & State Estimate Extrapolation \\
\hline$P_{k}(-)=\Phi_{k-1} P_{k-1}(+) \Phi_{k-1}^{T}+Q_{k-1}$ & Covariance Estimate Extrapolation \\
\hline$K_{k}=P_{k}(-) H_{k}^{T}\left[H_{k} P_{k}(-) H_{k}^{T}+R_{k}\right]^{-1}$ & Filter Gain Computation \\
\hline$\hat{x}_{k}(+)=\hat{x}_{k}(-)+K_{k}\left[z_{k}-H_{k} \hat{x}_{k}(-)\right]$ & State Estimate Update \\
\hline$P_{k}(+)=\left[P_{k}(-)^{-1}+H_{k}^{T} R_{k}^{-1} H_{k}\right]^{-1}$ & Covariance Estimate Update \\
\hline
\end{tabular}
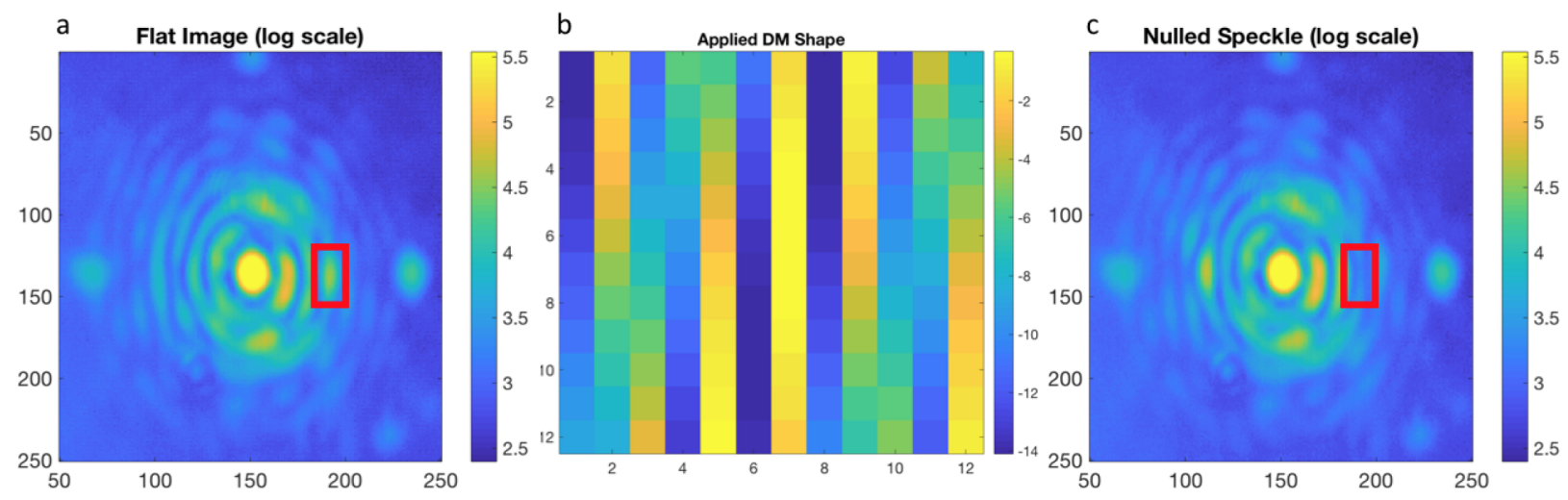

Figure 5. From left to right: a) an example image of the PSF with an injected speckle shown within the red box, b) the shape of the DM that creates the anti-speckle that best nulls the initial speckle, c) image in the science camera after the speckle is nulled

\subsection{Previous Results}

Below is a recap of the results from Xin et al. 2018, the experiments of which were conducted using the same laboratory setup.

Figure 6 shows the raw contrast readings through the fiber for speckle nulling both with and without the Kalman filter. Only the first 100 out of 500 iterations are shown, though the contrast trend continues in a similar fashion. Using all 500 iterations, a contrast improvement of $10 \%$ was found. ${ }^{5}$ 


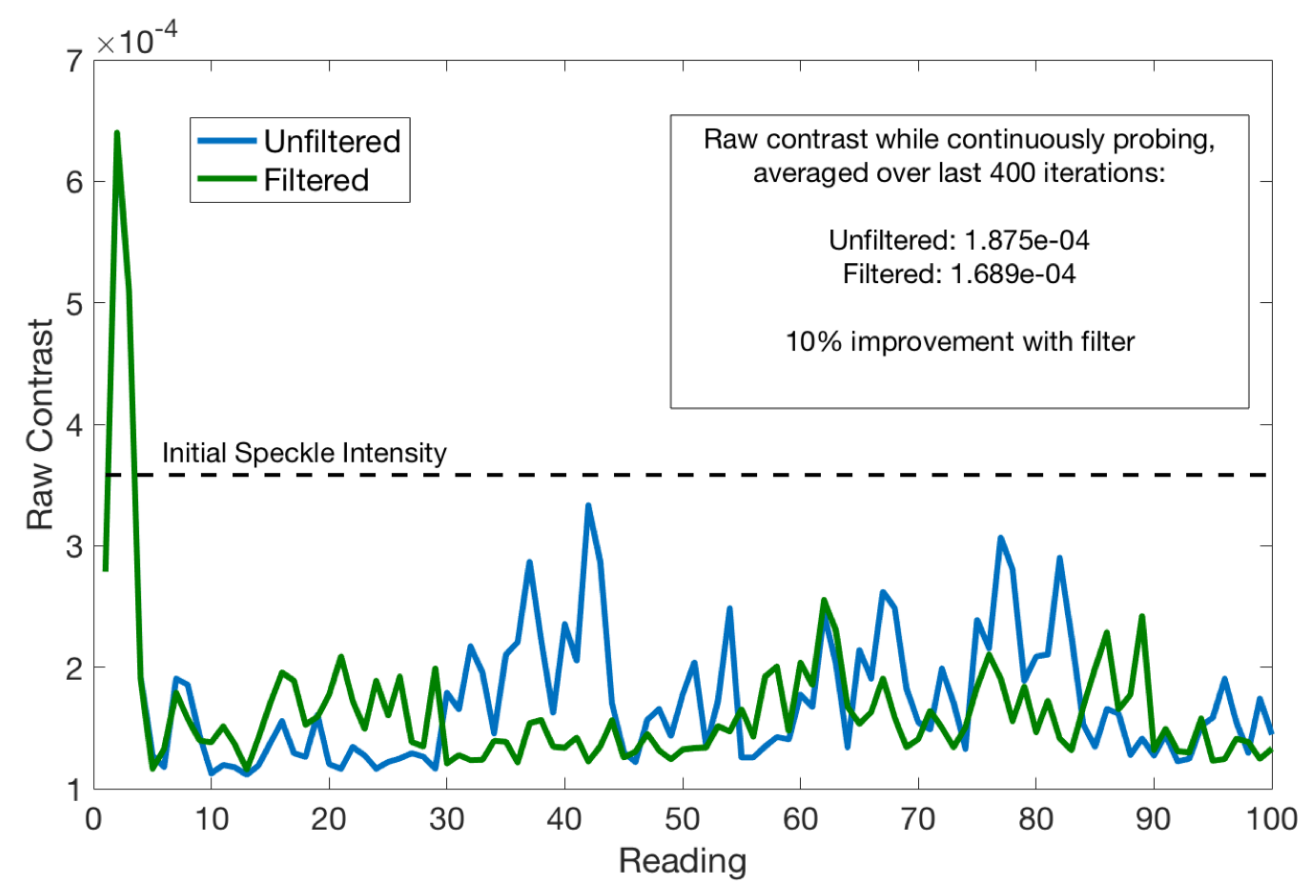

Figure 6. The raw contrast readings for speckle nulling both with and without the Kalman filter. For the filtered contrast, an improvement of $10 \%$ was found. ${ }^{5}$

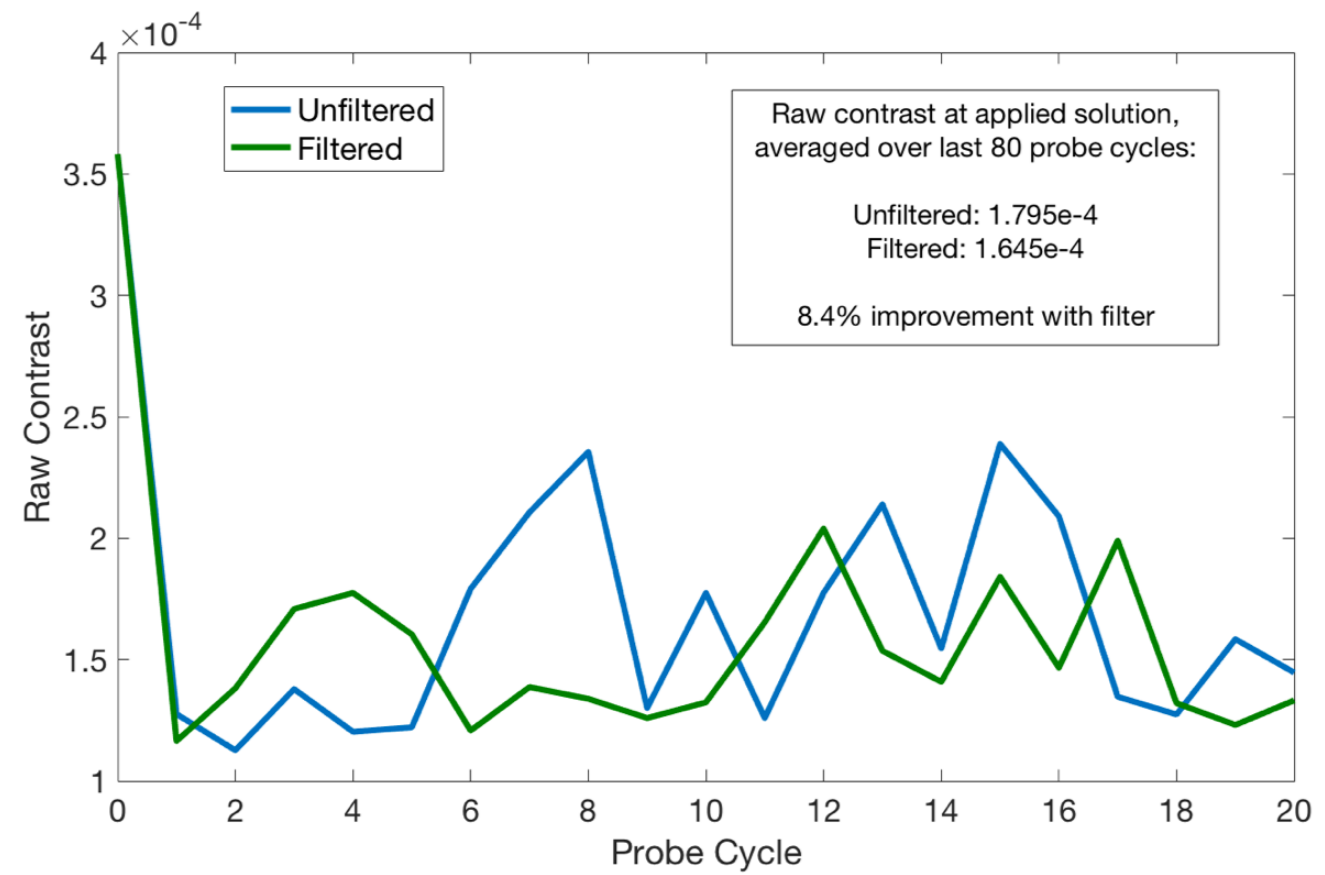

Figure 7. The raw contrast readings for speckle nulling both with and without the Kalman filter, with the solution applied to every 5 th reading of the data. For the filtered contrast, an improvement of $8.4 \%$ was found. ${ }^{5}$

Figure 7 is similar to figure 6 except that the contrast readings were taken only after the solution was applied in every 5 th reading of the data. The filter resulted in a $8.4 \%$ improvement in nulling. ${ }^{5}$

The quantitative improvement was a very promising demonstration of the contrast gains possible with the 
Kalman filter. However, it was still somewhat unclear when the filter resulted in greater gains, and it became a new goal to show that the filter did indeed help with stability, particularly in the case of small disturbances.

\subsection{Updated Results}

For these updated results, the filter architecture was improved, most notably through the improvement of estimates for the parameters of the system. In order to figure out the best possible parameters for the guesses for the filter, a survey of both the measurement noise (present in both the measurement of the amplitude and phase of the sinusoid of best fit for the speckle) and process noise was conducted. After the determination of the parameters that resulted in the greatest contrast improvements (both with and without the filter), speckle nulling with and without the Kalman filter was run with the best parameters. Below are the results of two typical cases of runs, showing the results both with and without the filter.

Figure 9 was very promising in that it showed that the filter did indeed help noticeably with contrast stability due to the more stable past control history. Though it is unclear what specific disturbances might have been behind these particular contrast degradation, known sources of disturbances in the laboratory include turbulence, vibrations, and measurements noise. It was also promising that in stable cases, as shown in figure ref StableCase where there are very little disturbances to the system and thus very little variation in the raw contrast, that the contrast achieved with the filter was essentially the same as that with the measurement. This means that even at worst, the filtered nulling does not perform any worse than the unfiltered nulling.

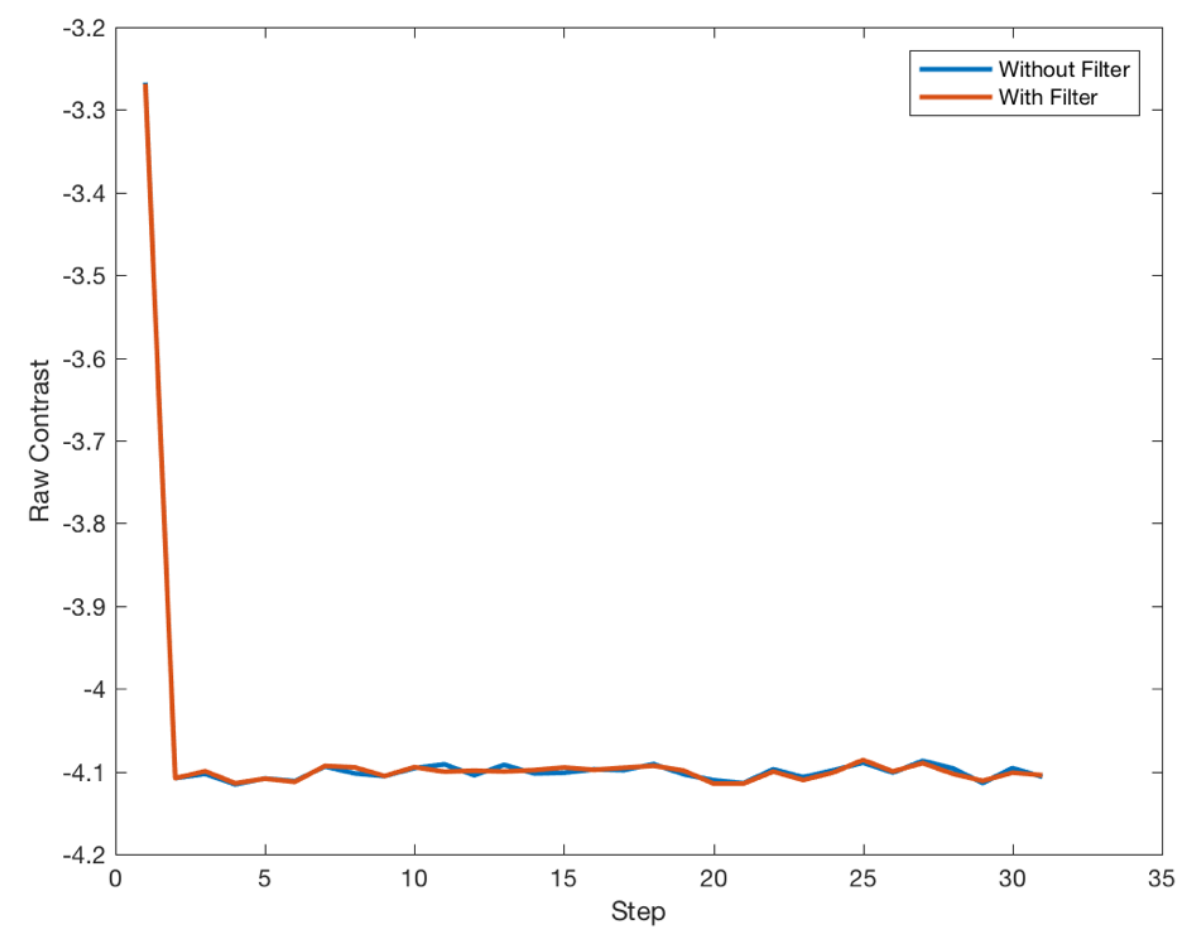

Figure 8. An example of a relatively stable case, in which both the contrast with and without the filter does not vary greatly. 


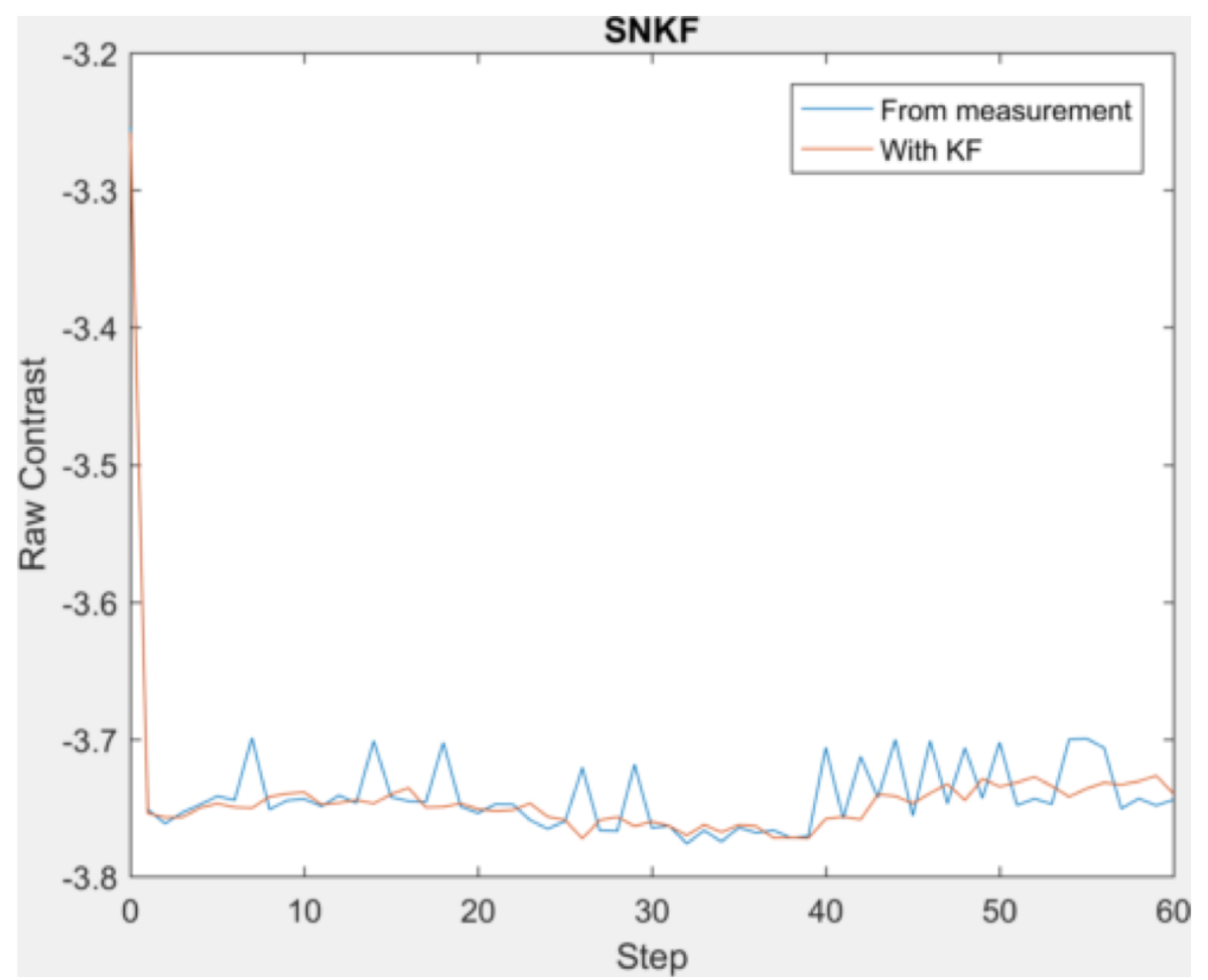

Figure 9. An example of an unstable case, notably with small disturbances for the unfiltered measurements in which the contrast degrades. During these disturbances, the filtered measurements are shown to also not vary much.

\subsection{Conclusions and Further Work}

We have shown that the Kalman filter estimator does indeed help increase stability especially in the case of small disturbances. Even in cases where there is not significant improvement with the filter, the contrast with the filter is not worse than that without the filter. In the future, the filter estimation process could also be used as a framework for predictive atmospheric control. The residuals could be used to fit a simple, one-mode model of the atmosphere's effects at the fiber.

Next steps would include introducing more noise sources. This would include measurement noise (artificial detector noise), artificially induced DM jitter, and turbulence simulation. The jitter could be implemented by adding Gaussian noise to the movement of the DM, and turbulence can be simulated by running pre-determined sequences of patterns onto the actuators while it is also running the speckle nulling algorithm. There could also be a finer probe for the estimate parameter values and it is also possible to apply multiple sinusoids. With those two steps, a more precise null could possibly be achieved.

As the speckle was also fairly faint and close to the nulling floor, it is possible that the filter could show more significant improvements with a brighter speckle, which would be fairly simple to test as well.

All tests were conducted on the transmissive testbed of HCST. With the reflective testbed of HCST, a new fiber injection unit will be implemented. With a more stable system, better calibrated disturbances can be added to the system, and it could be an ideal testbed on which to carry out next steps and also confirm the gains in stability found with the Kalman filter estimators.

\section{REFERENCES}

[1] Mawet, D., Ruane, G., Xuan, W., Echeverri, D., Klimovich, N., Randolph, M., Fucik, J., Wallace, J. K., Wang, J., Vasisht, G., and et al., "Observing exoplanets with high-dispersion coronagraphy. ii. demonstration of an active single-mode fiber injection unit," The Astrophysical Journal 838(2), 92 (2017). 
[2] Delorme, J. R., Mawet, D., Fucik, J., Wallace, J. K., Ruane, G., Jovanovic, N., Klimovich, N. S., Llop Sayson, J. D., Zhang, R., Xin, Y., Riddle, R., Dekany, R., Wang, J., Choquet, E., Xuan, W., Echeverri, D., Randolph, M., Vasisht, G., and Mennesson, B., "High-contrast spectroscopy testbed for segmented telescopes," Proc. SPIE 10400, 104000X (2018).

[3] Malbet, F., Yu, J. W., and Shao, M., "High-dynamic-range imaging using a deformable mirror for space coronography," Publications of the Astronomical Society of the Pacific 107, 386 (1995).

[4] Groff, T. D. and Kasdin, N. J., "Kalman filtering techniques for focal plane electric field estimation," Journal of the Optical Society of America A 30(1), 128 (2012).

[5] Xin, Y., Klimovich, N., Mawet, D., Ruane, G., Delorme, J.-R., Jovanovic, N., and Sayson, J. D. L., "Demonstration of a speckle nulling algorithm and kalman filter estimator with a fiber injection unit for observing exoplanets with high-dispersion coronagraphy," Adaptive Optics Systems VI (2018). 